Supporting Information

\title{
Minimalization of Metallic Pd Formation in Suzuki Reaction with a Solid-State Organometallic Catalyst
}

\author{
Bowen Li, and Hua Chun Zeng* \\ Department of Chemical and Biomolecular Engineering, Faculty of Engineering, National \\ University of Singapore, 10 Kent Ridge Crescent, Singapore 119260 \\ *Email: chezhc@nus.edu.sg
}

\section{Table of Contents}

Supporting Figures (Figure S1 to Figure S21) ......................... Pages S-2 to S-18

Supporting Tables (Table S1 to Table S8)............................ Pages S-19 to S-26 


\section{Supporting Figures}
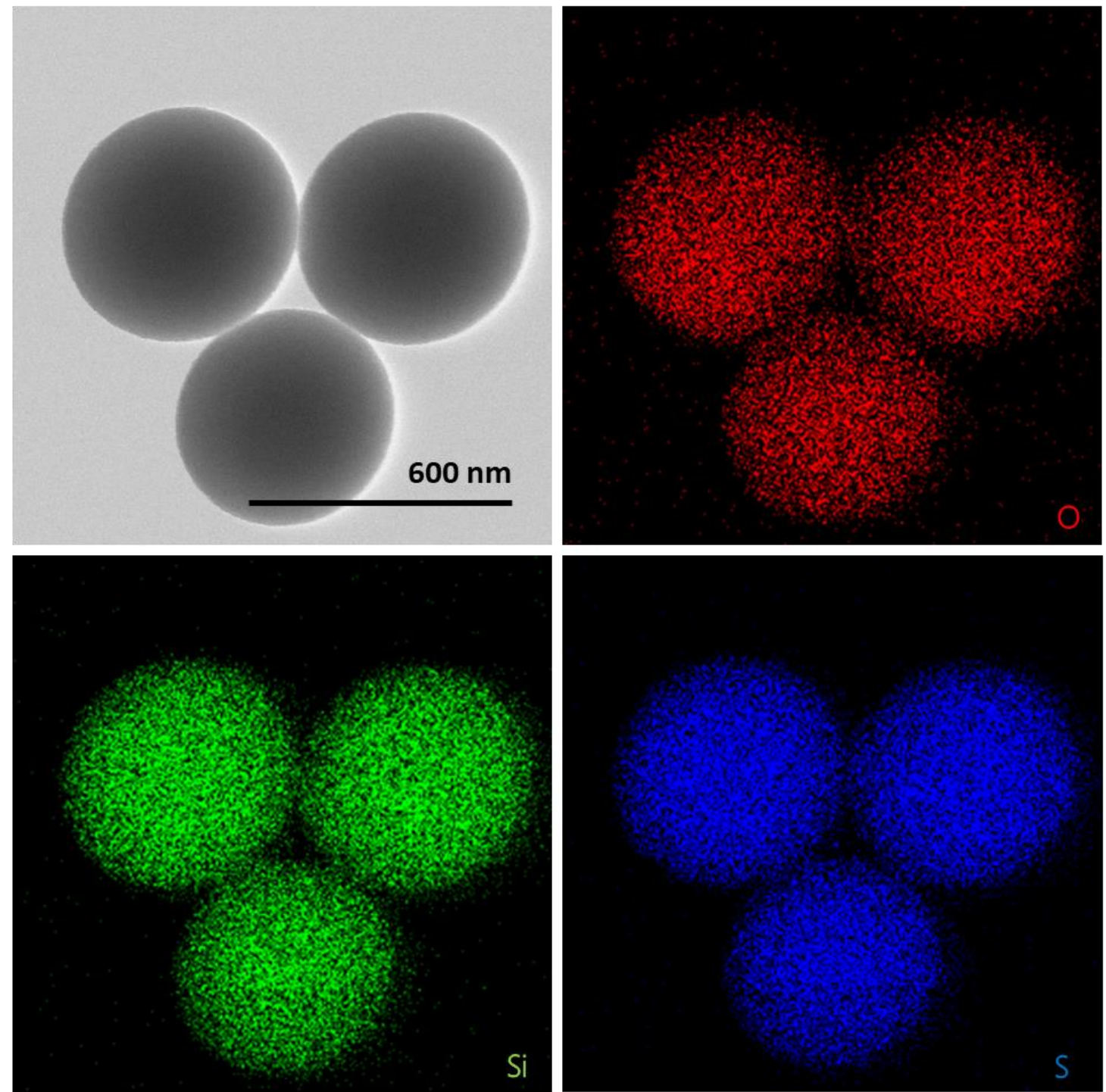

Figure S1. STEM image and corresponding EDX mappings of MPOS spheres. 

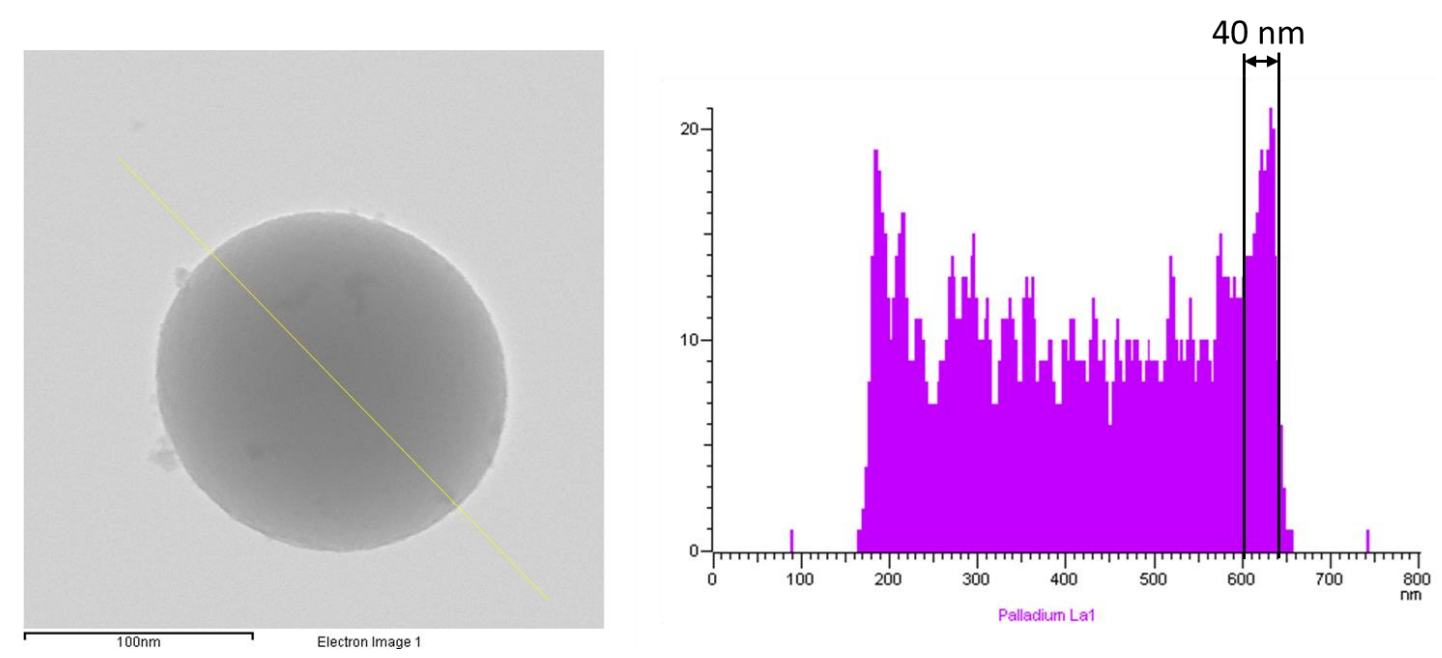

Figure S2. EDX line scan of Pd for a MPOS-Pd sphere. The dual-peak shaped line scan indicates that the immobilized Pd ions were concentrated near MPOS surface. The width of Pd line scan peak has revealed that the immobilized Pd ions were concentrated within a surface shell of $40 \mathrm{~nm}$ thickness.

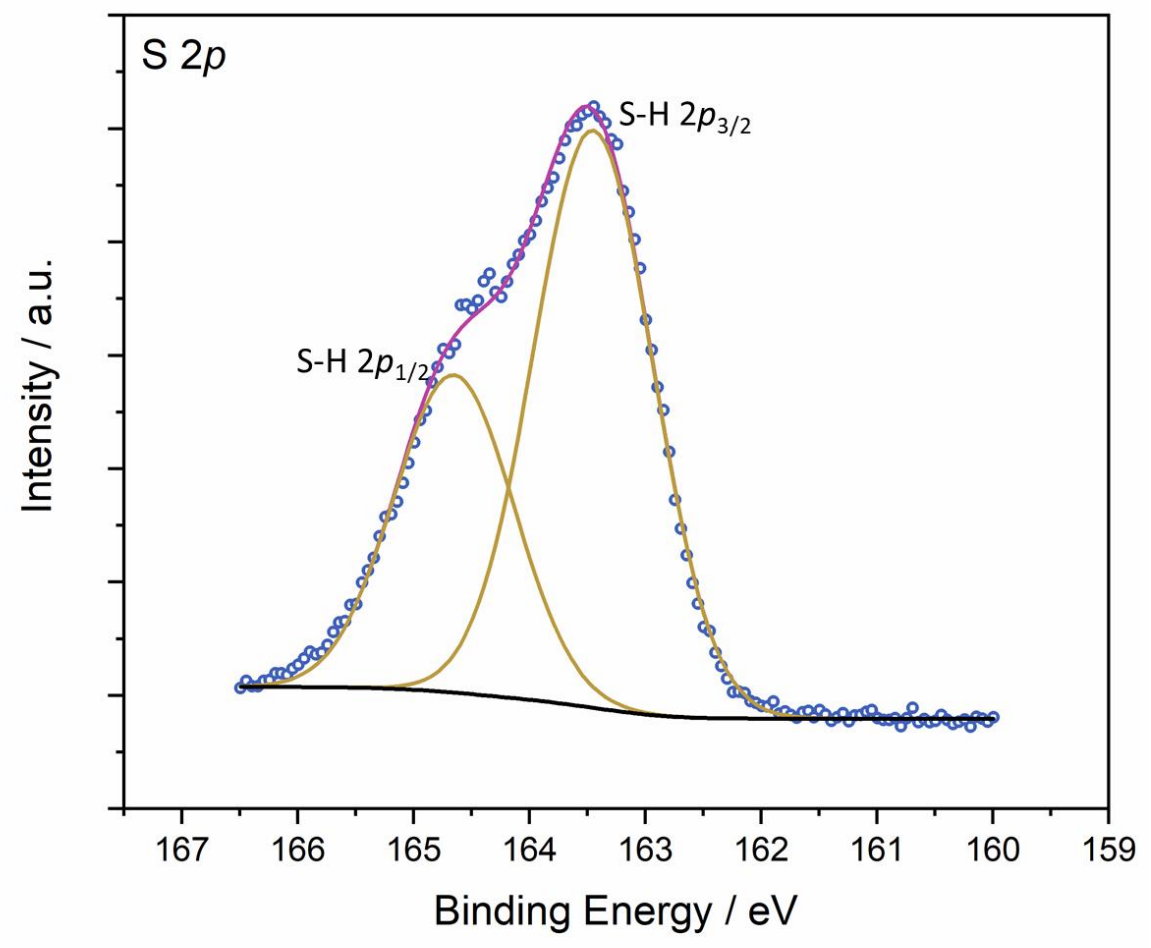

Figure S3. S $2 p$ XPS spectrum of MPOS spheres. The two peaks at $165.5 \mathrm{eV}$ and $164.7 \mathrm{eV}$ are assigned to $2 p_{3 / 2}$ and $2 p_{1 / 2}$ of sulfur in R-S-H thiol group respectively. 

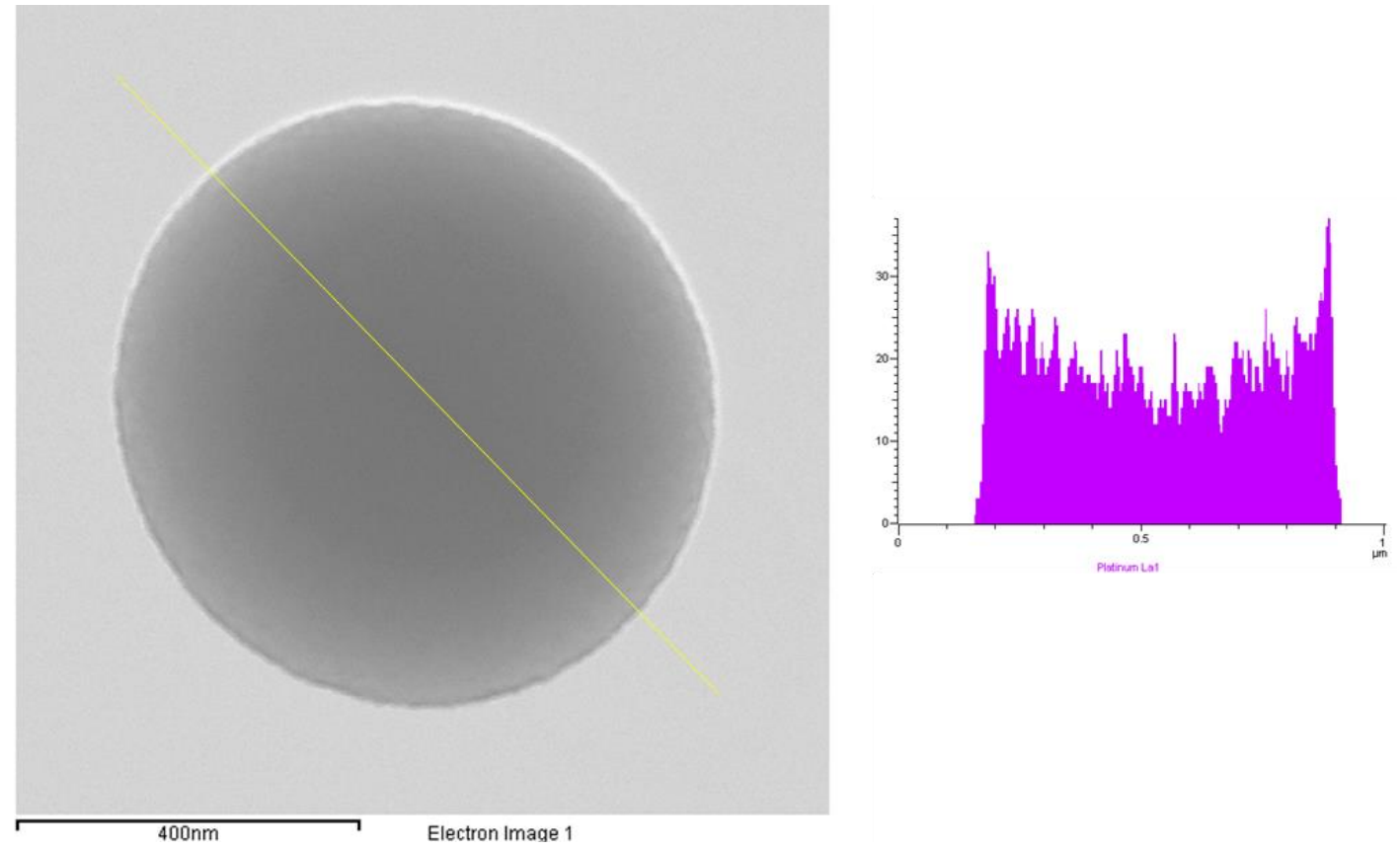

Figure S4. EDX line scan of Pt for a MPOS-Pt sphere. The same dual-peak shape has revealed similar distribution of $\mathrm{Pt}$ as compared to $\mathrm{Pd}$.

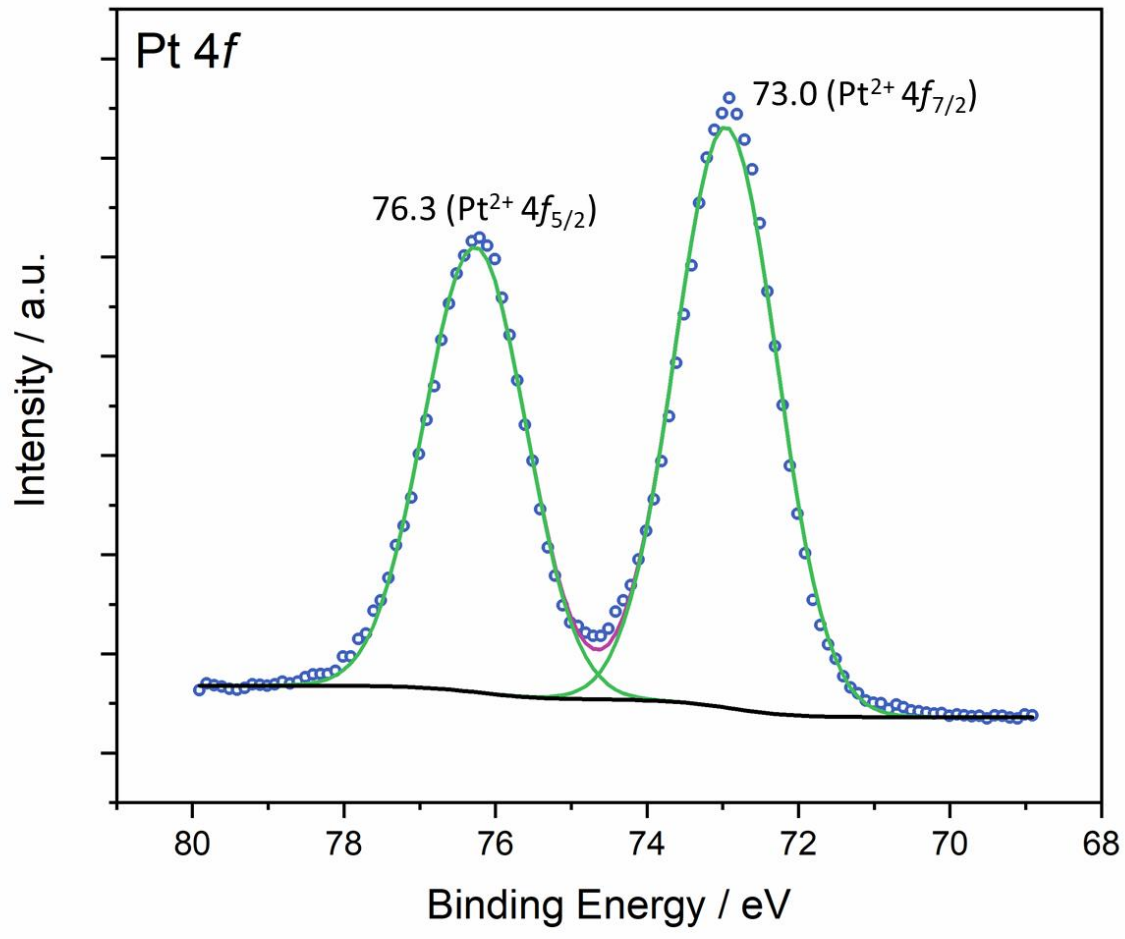

Figure S5. Pt $4 f$ XPS spectrum of MPOS-Pt spheres. The two peaks at $73.0 \mathrm{eV}$ and $76.3 \mathrm{eV}$ could be assigned to $4 f_{7 / 2}$ and $4 f_{5 / 2}$ of $\mathrm{Pt}^{2+}$ respectively. 

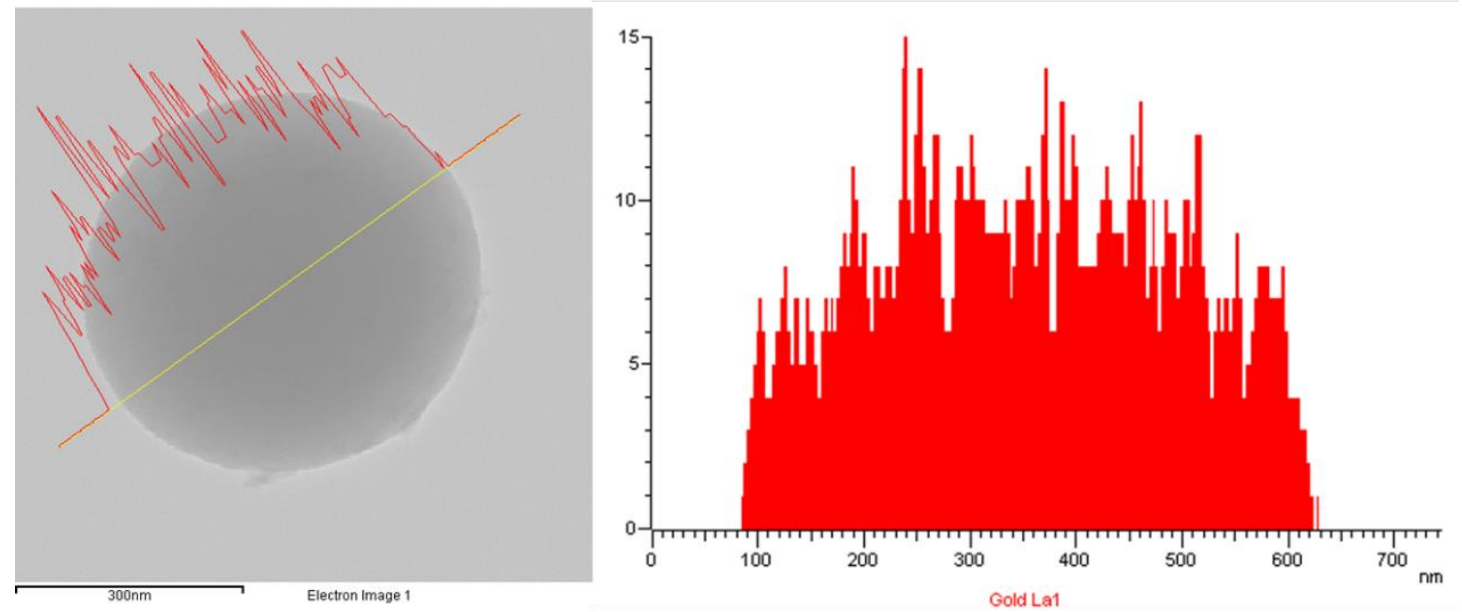

Figure S6. EDX line scan of $\mathrm{Au}$ for a MPOS-Au sphere. The shape of Au line scan has suggested homogeneous distribution of $\mathrm{Au}$ across the entire sphere.

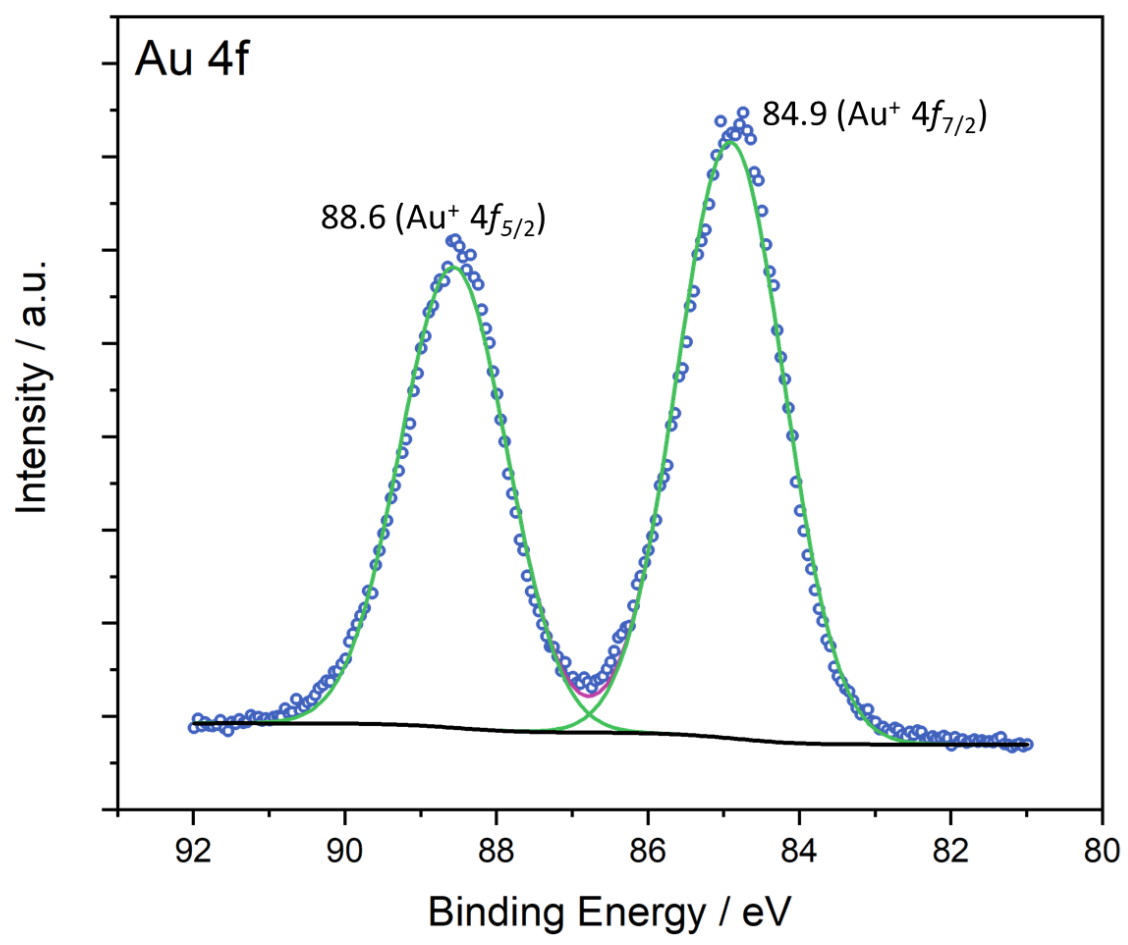

Figure S7. Au $4 f$ XPS spectrum of MPOS-Au spheres. The two peaks at $84.9 \mathrm{eV}$ and $88.6 \mathrm{eV}$ for $\mathrm{Au} 4 f$ spectrum are assigned to $4 f_{7 / 2}$ and $4 f_{5 / 2}$ of $\mathrm{Au}^{+}$respectively. 


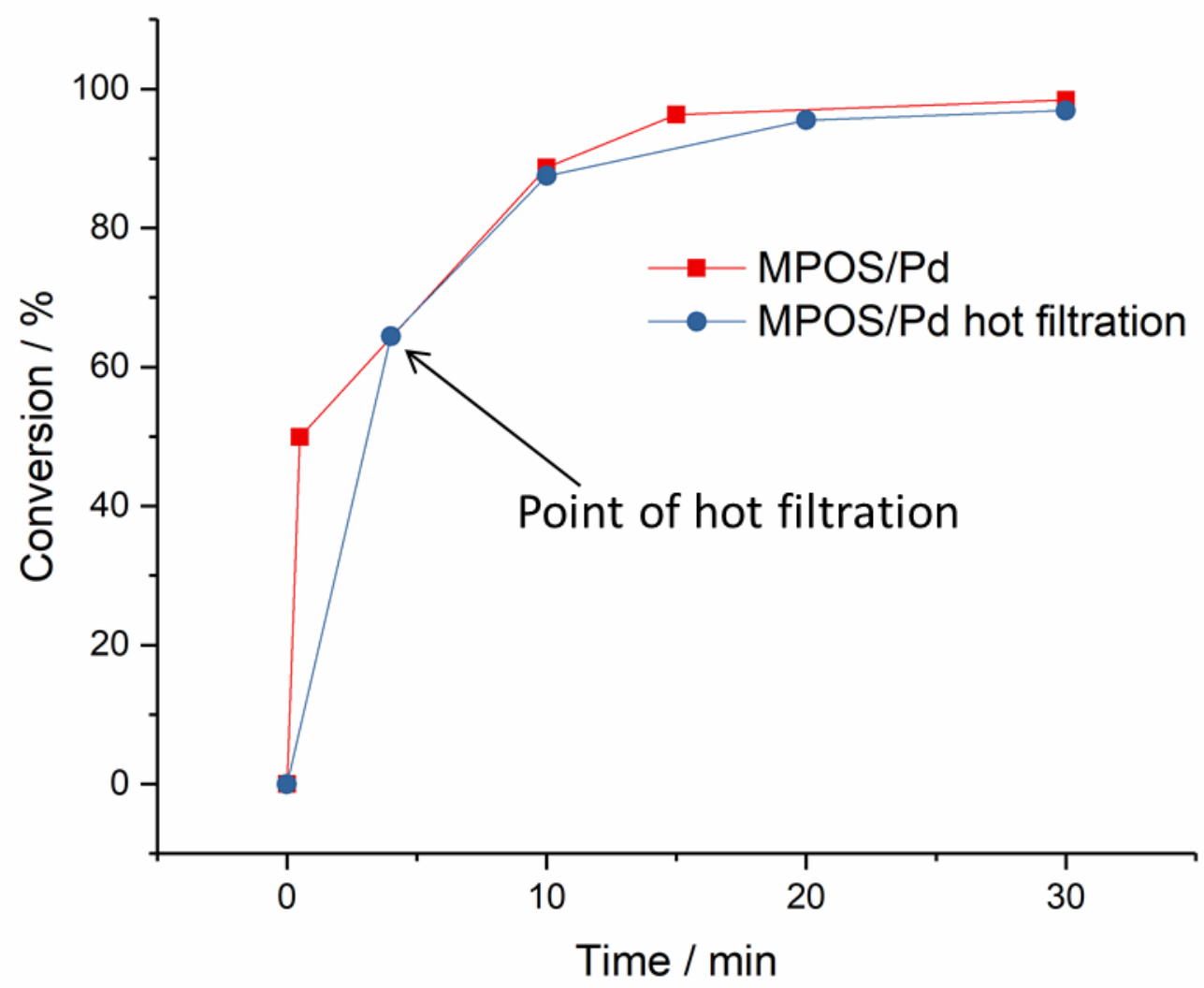

Figure S8. Conversion rate comparison between MPOS-Pd and MPOS-Pd hot filtrate for Suzuki-Miyaura reaction at $80{ }^{\circ} \mathrm{C}$ with different reaction times. 

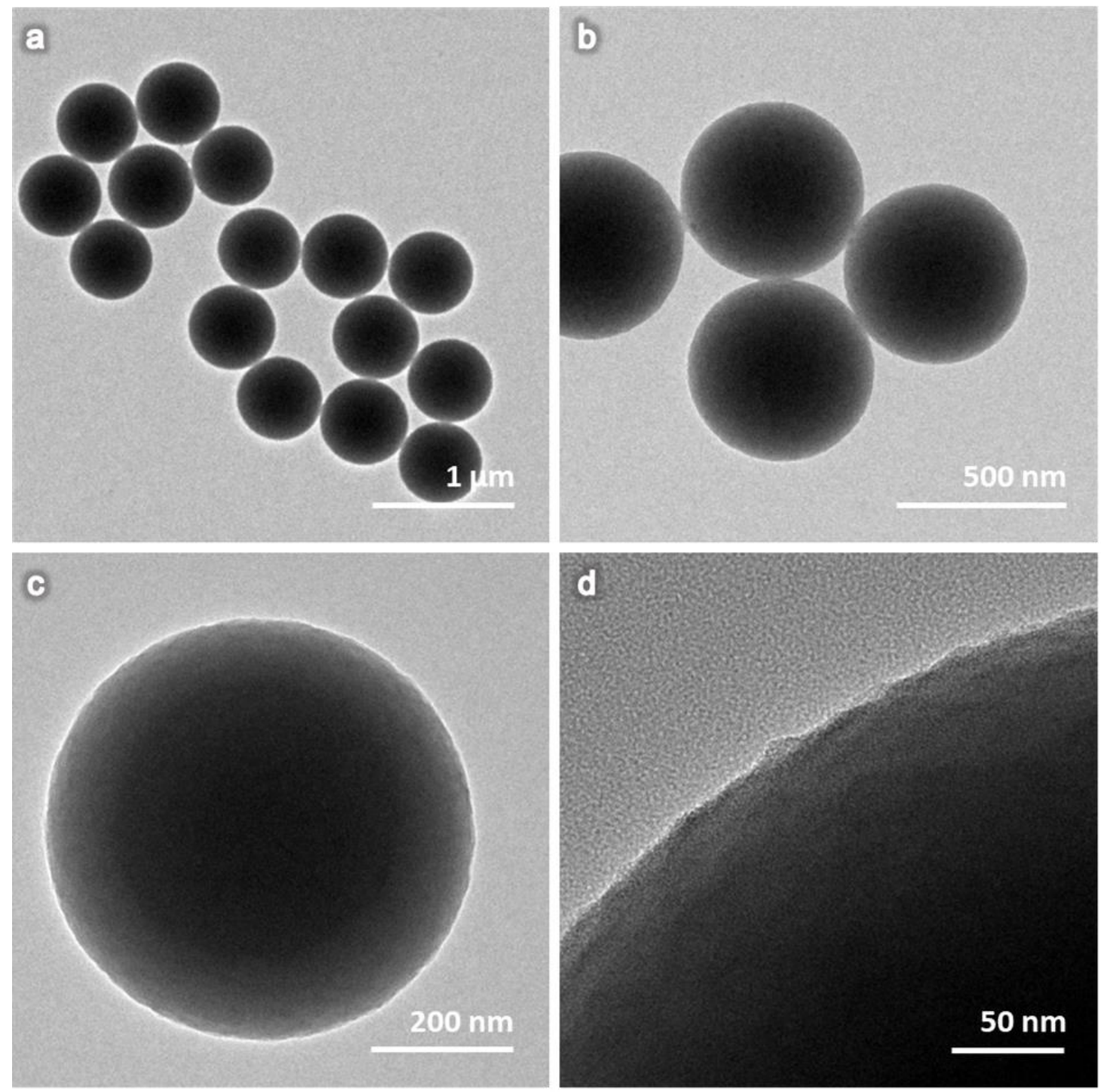

Figure S9. Representative TEM images (at different magnifications) of spent MPOS-Pd catalyst after 1 cycle of Suzuki-Miyaura reaction at $80{ }^{\circ} \mathrm{C}$ for $30 \mathrm{~min}$. 

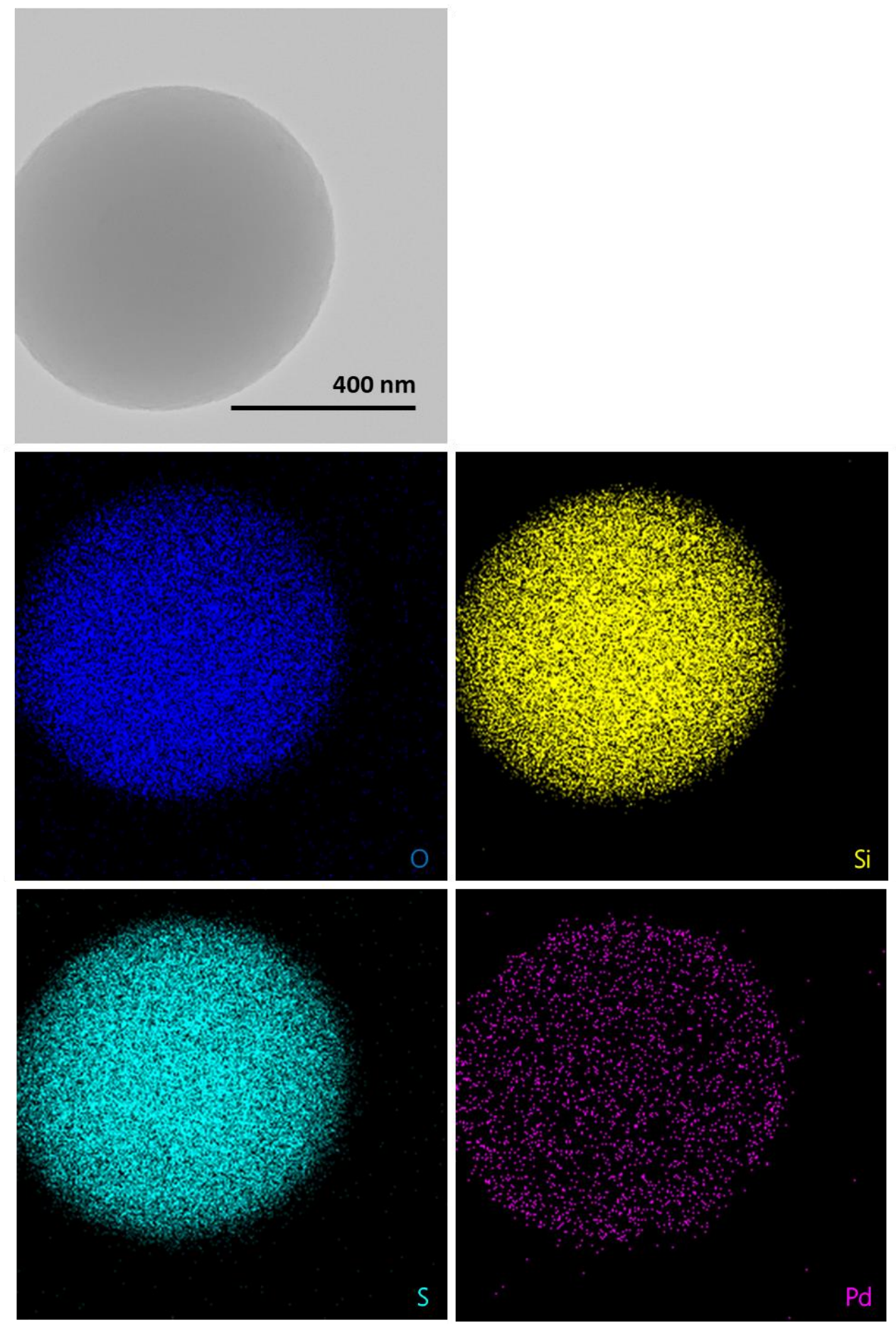

Figure S10. STEM image and corresponding EDX mappings of spent MPOS-Pd catalyst after 1 cycle of Suzuki-Miyaura reaction at $80^{\circ} \mathrm{C}$ for $30 \mathrm{~min}$. 

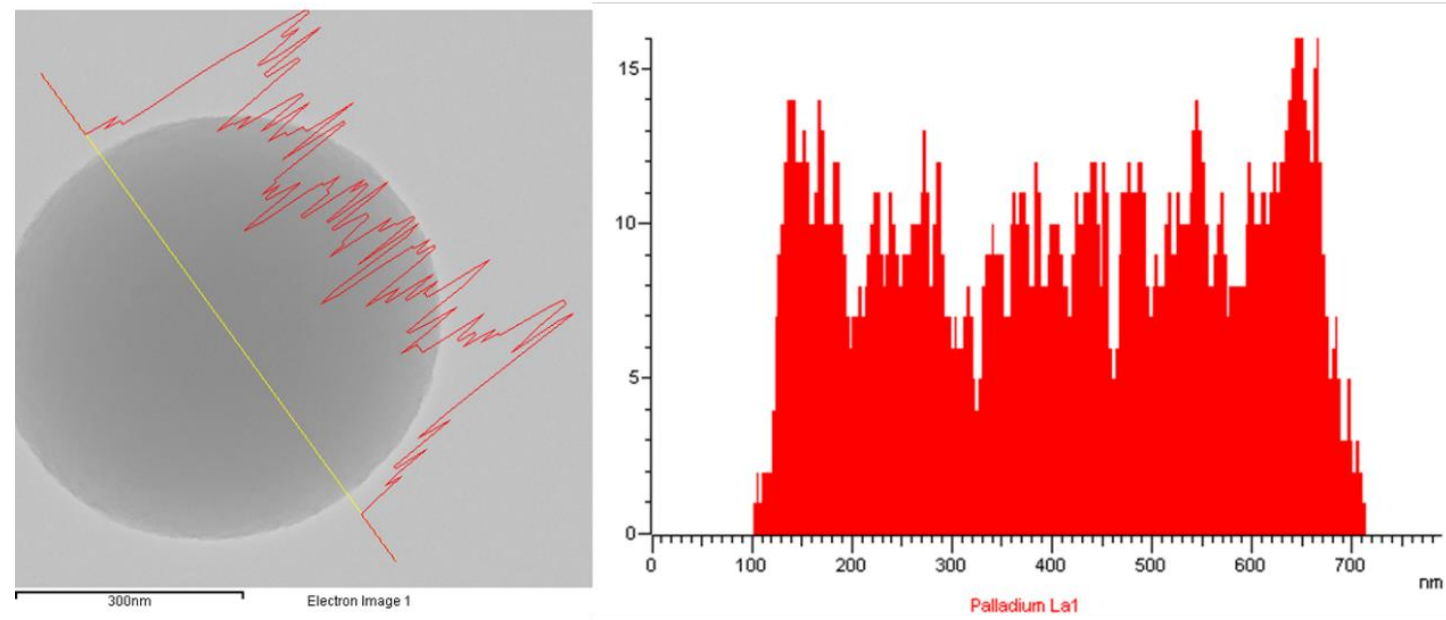

Figure S11. EDX line scan of Pd for spent MPOS-Pd catalyst after 1 cycle of Suzuki-Miyaura reaction at $80^{\circ} \mathrm{C}$ for $30 \mathrm{~min}$. 

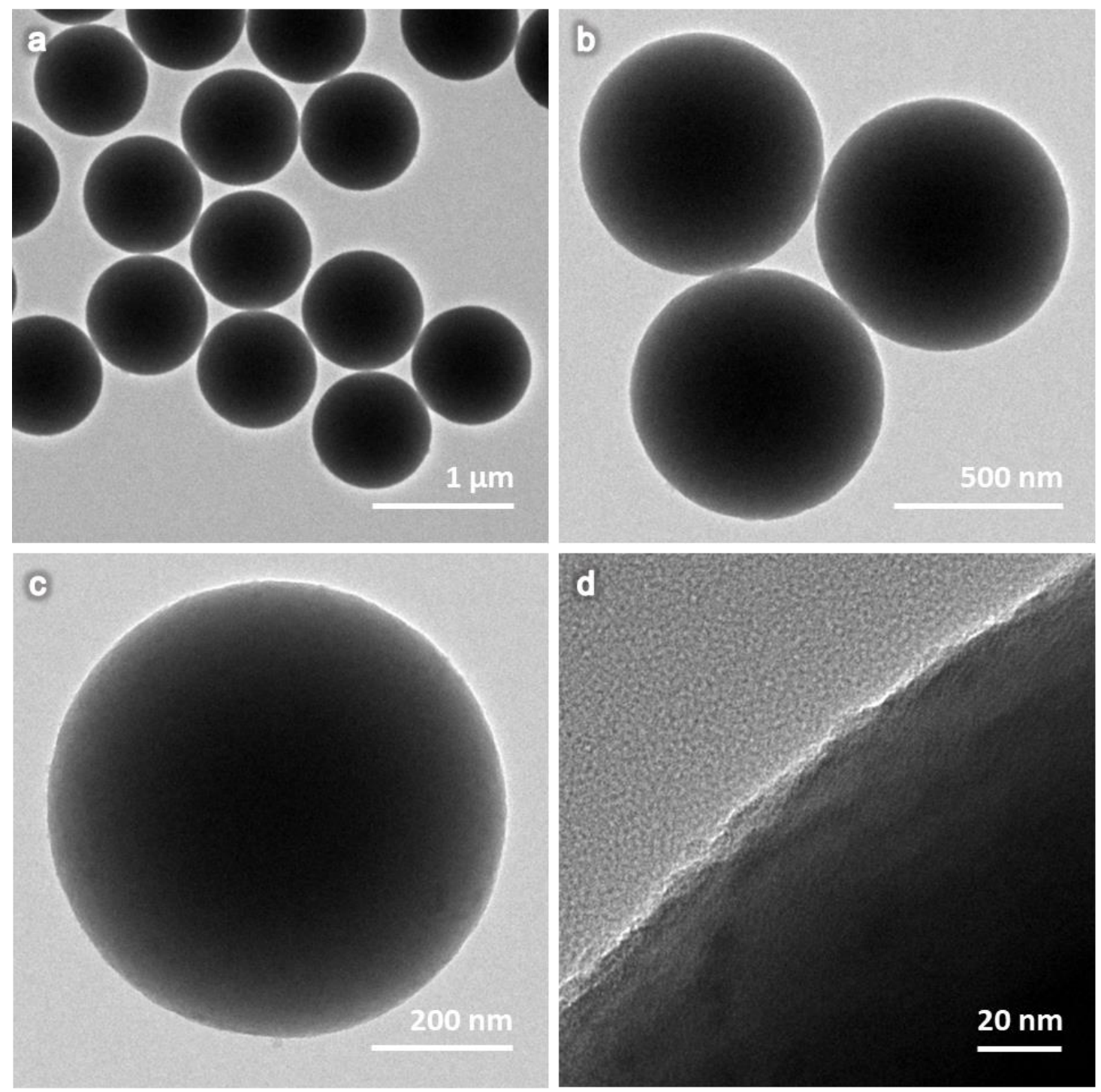

Figure S12. Representative TEM images (at different magnifications) of spent MPOS-Pd catalyst after 5 cycles of Suzuki-Miyaura reaction at $80{ }^{\circ} \mathrm{C}$ for $30 \mathrm{~min}$. 

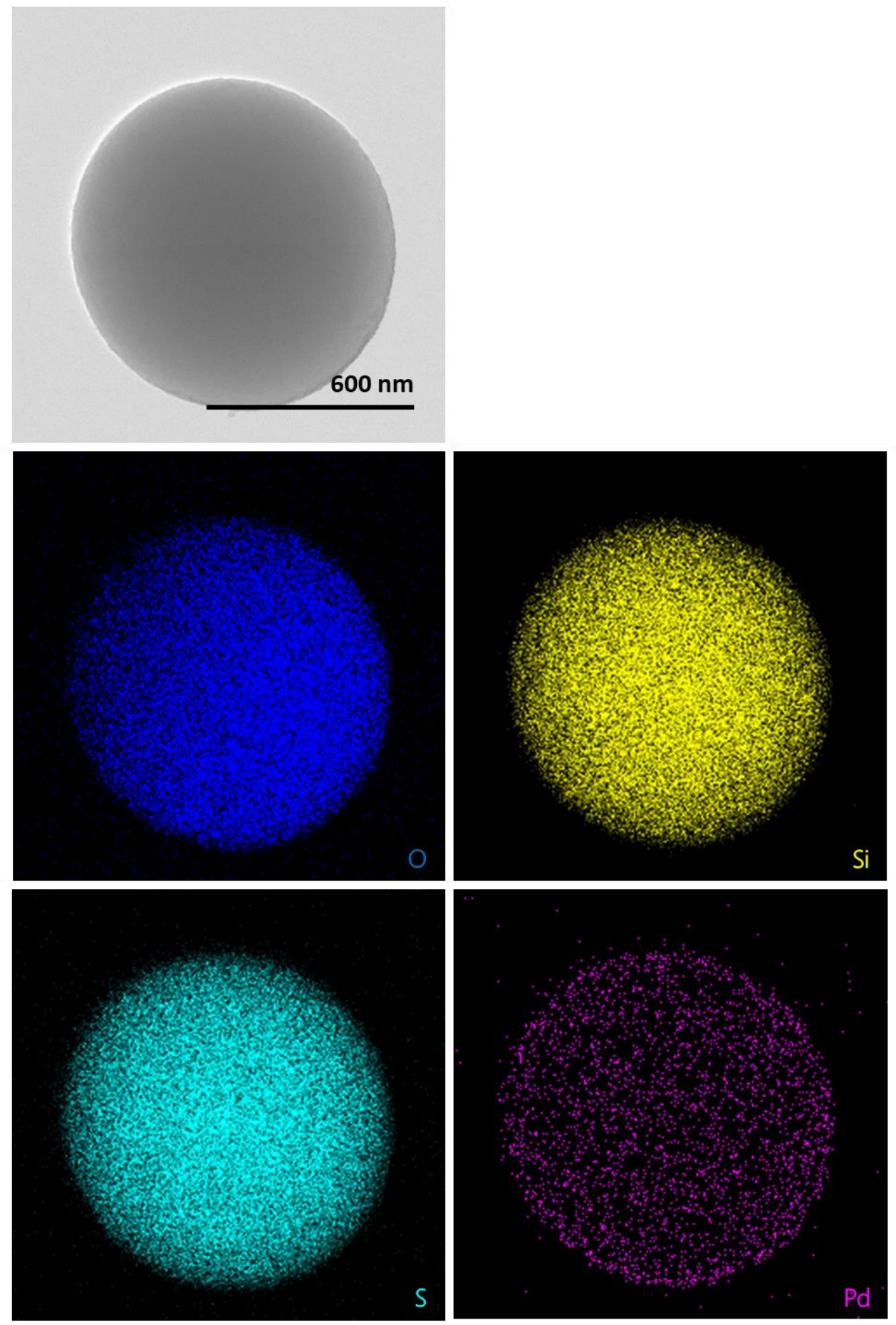

Figure S13. STEM image and corresponding EDX mappings of spent MPOS-Pd catalyst after 5 cycles of Suzuki-Miyaura reaction at $80^{\circ} \mathrm{C}$ for $30 \mathrm{~min}$. 

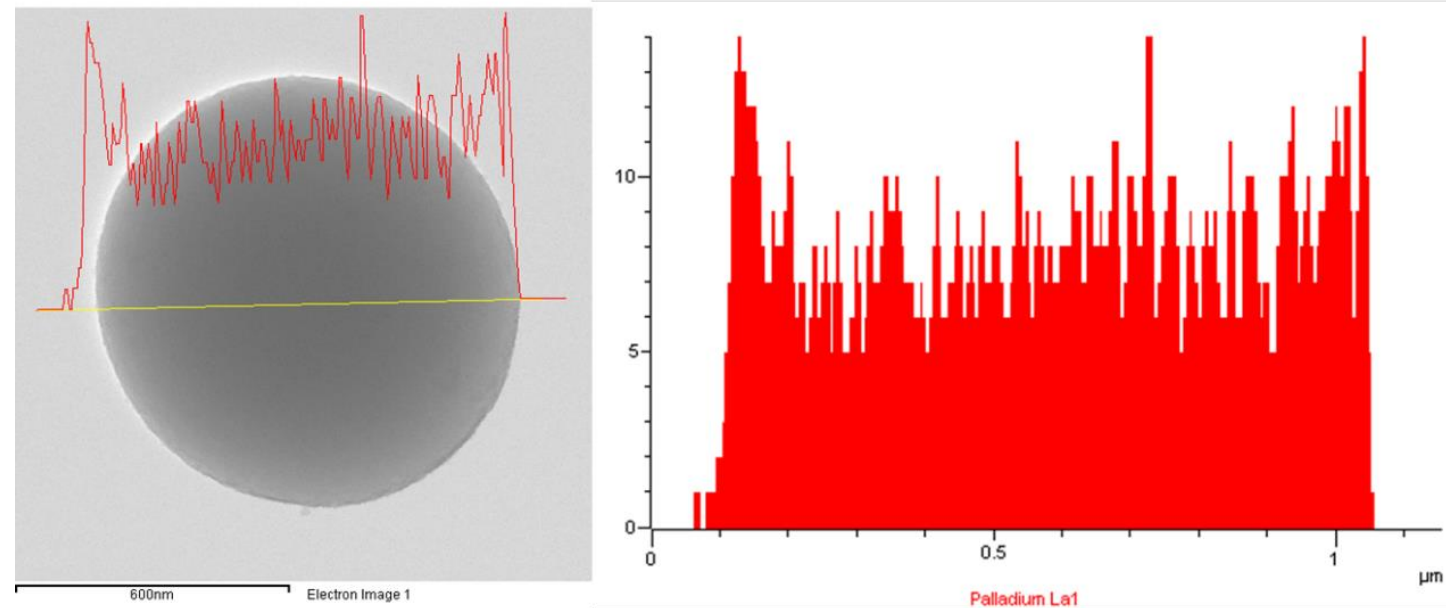

Figure S14. EDX line scan of Pd for spent MPOS-Pd catalyst after 5 cycles of Suzuki-Miyaura reaction at $80{ }^{\circ} \mathrm{C}$ for $30 \mathrm{~min}$. 

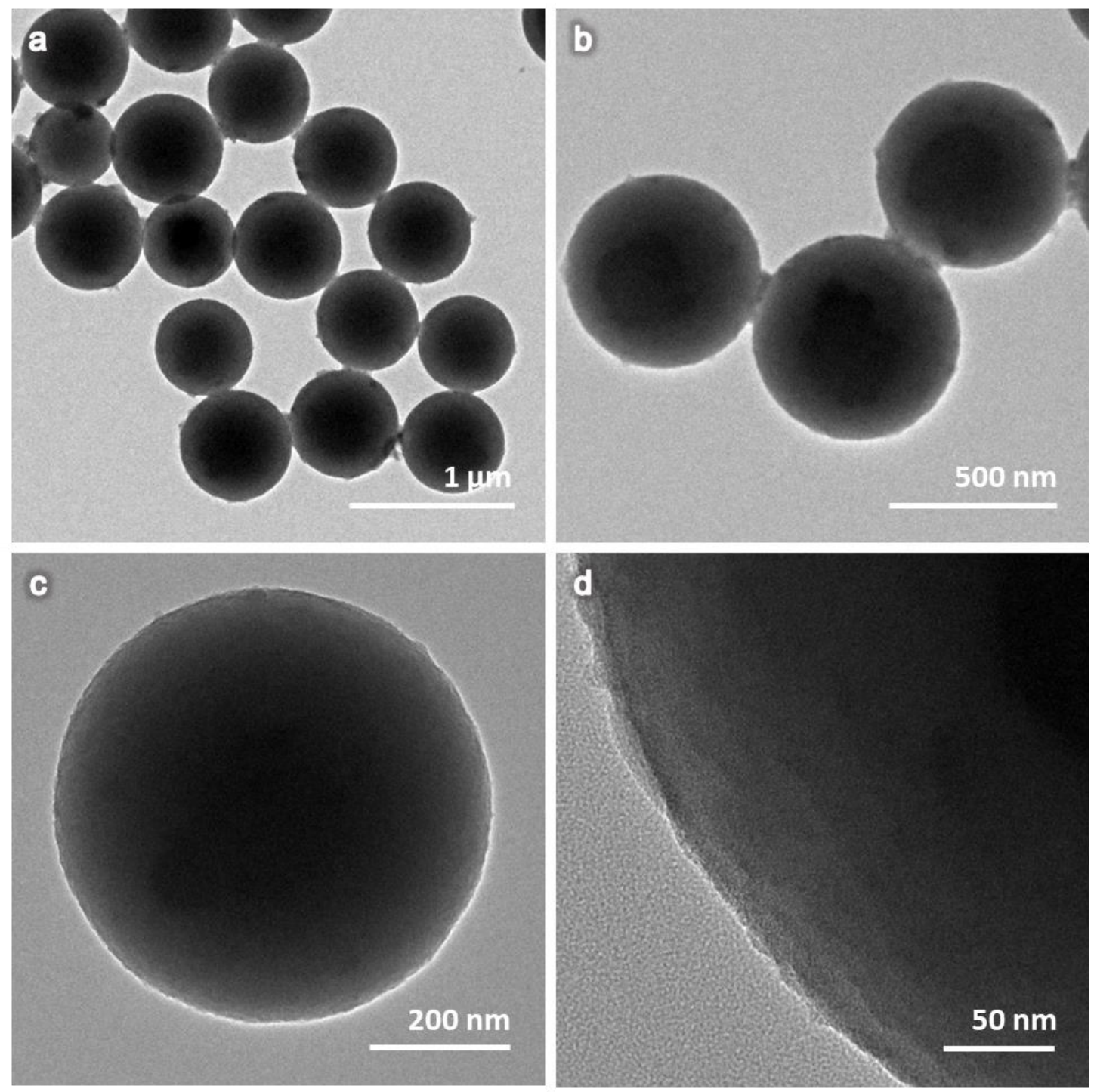

Figure S15. Representative TEM images (at different magnifications) of spent MPOS-Pd catalyst after 5 cycles of Suzuki-Miyaura reaction at $80{ }^{\circ} \mathrm{C}$ for $60 \mathrm{~min}$. 

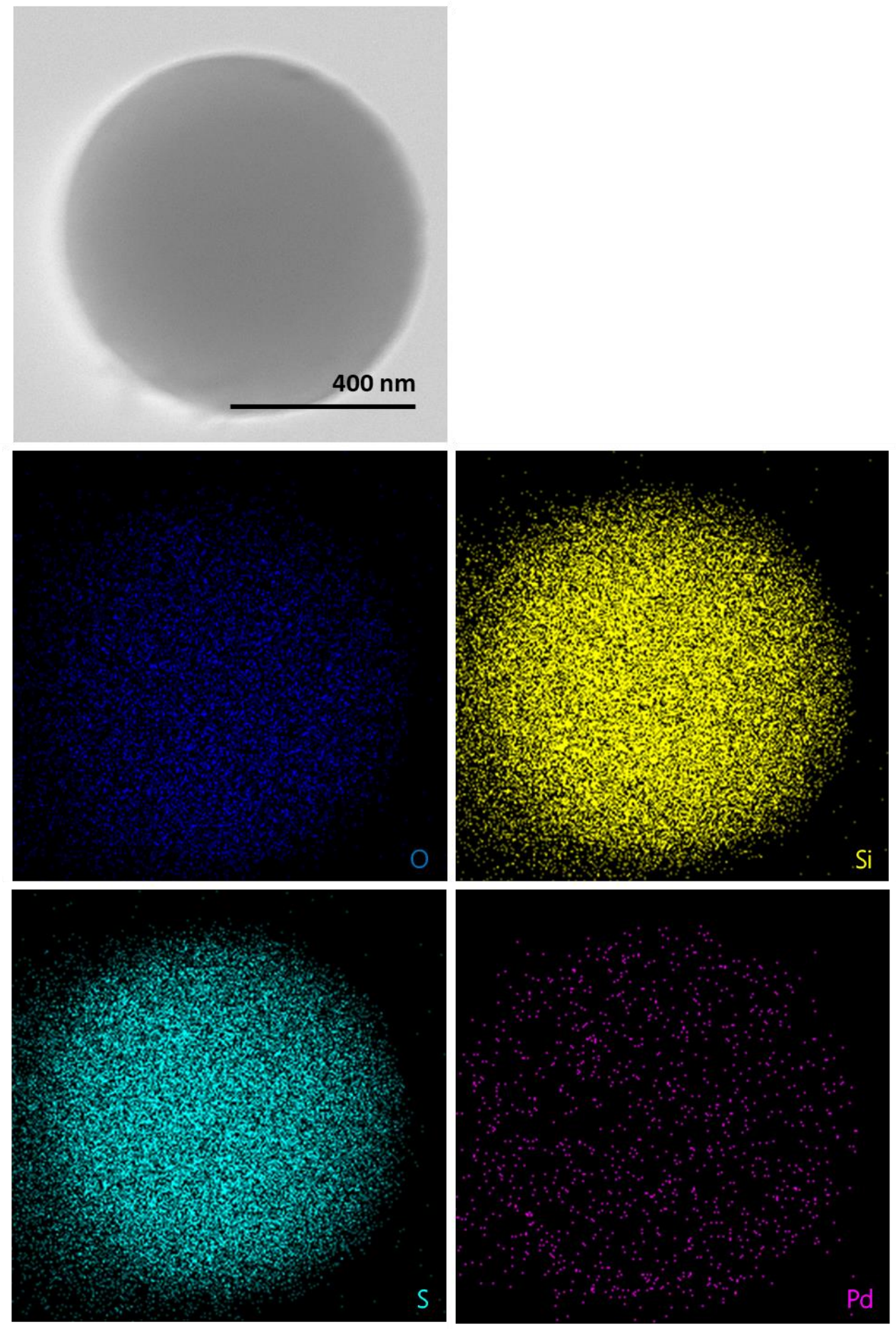

Figure S16. STEM image and corresponding EDX mappings of spent MPOS-Pd catalyst after 5 cycles of Suzuki-Miyaura reaction at $80^{\circ} \mathrm{C}$ for $60 \mathrm{~min}$. 

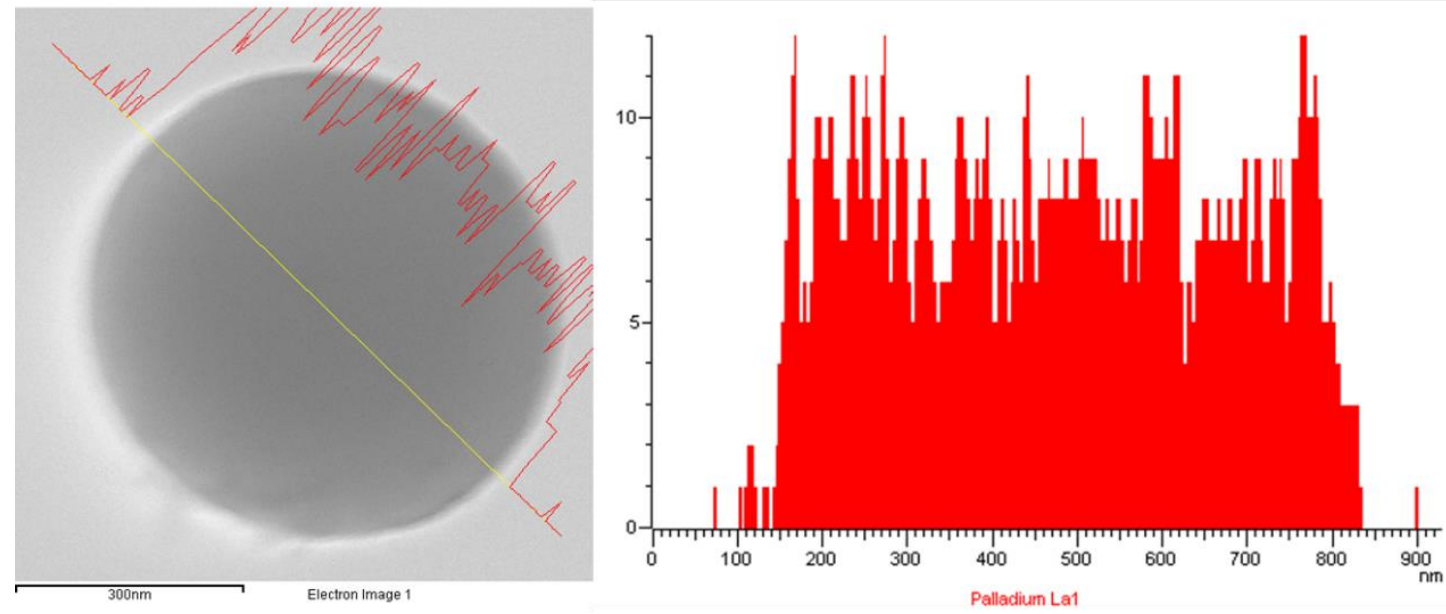

Figure S17. EDX line scan of Pd for spent MPOS-Pd catalyst after 5 cycles of Suzuki-Miyaura reaction at $80^{\circ} \mathrm{C}$ for $60 \mathrm{~min}$. 

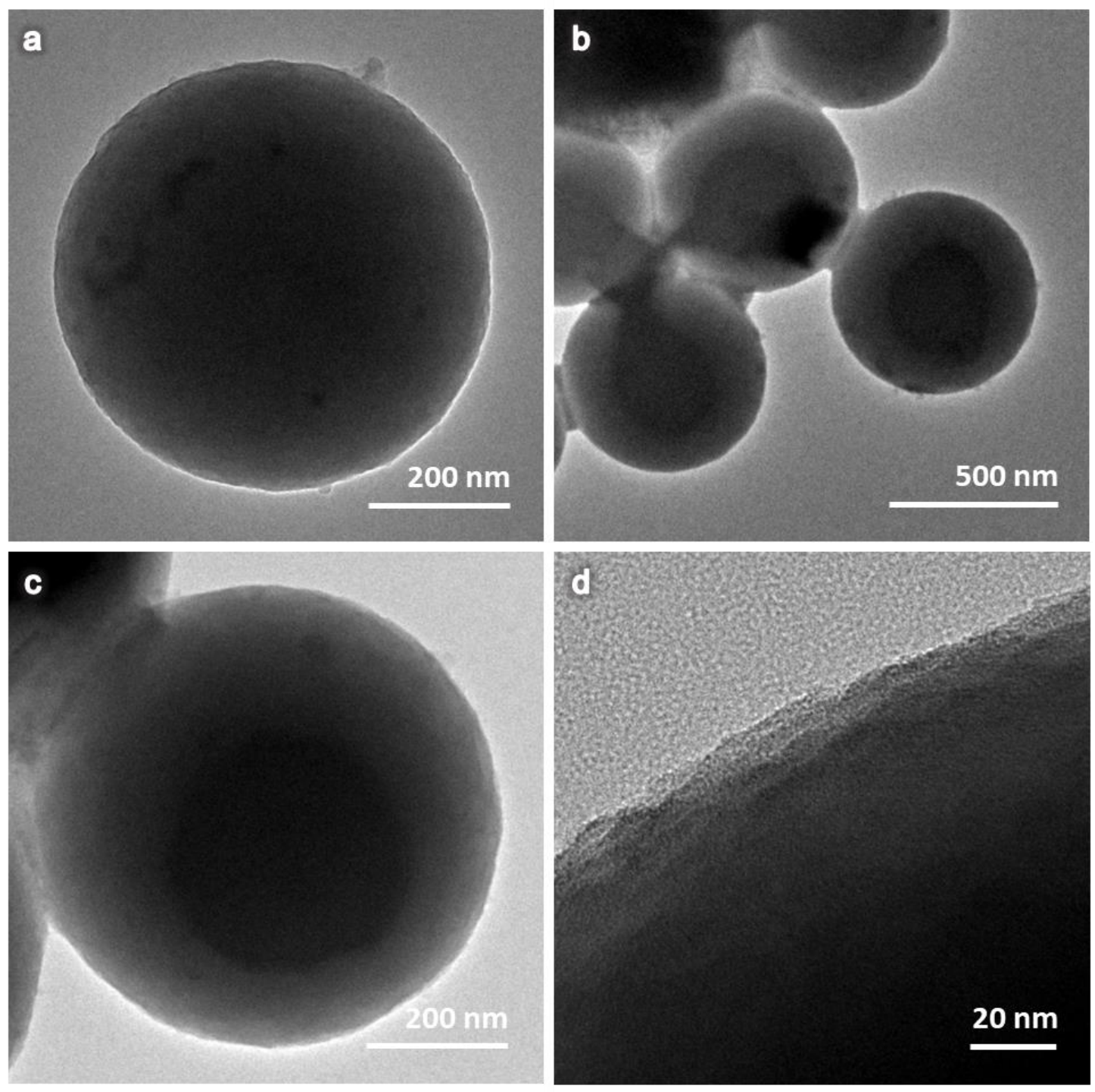

Figure S18. Representative TEM images (at different magnifications) of spent MPOS-Pd catalyst after 5 cycles of Suzuki-Miyaura reaction at $50{ }^{\circ} \mathrm{C}$ for $60 \mathrm{~min}$. 

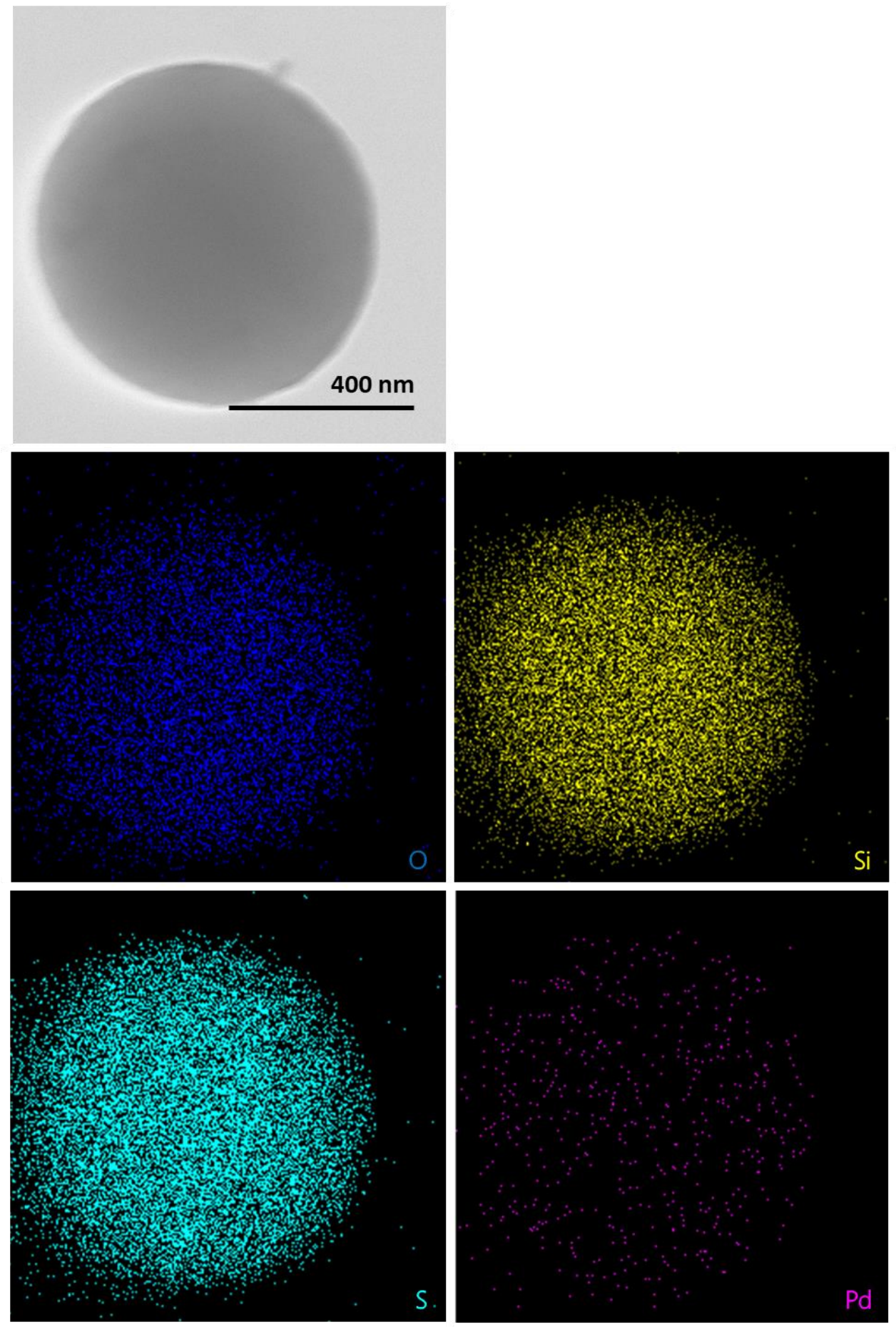

Figure S19. STEM image and corresponding EDX mappings of spent MPOS-Pd catalyst after 5 cycles of Suzuki-Miyaura reaction at $50^{\circ} \mathrm{C}$ for $60 \mathrm{~min}$. 

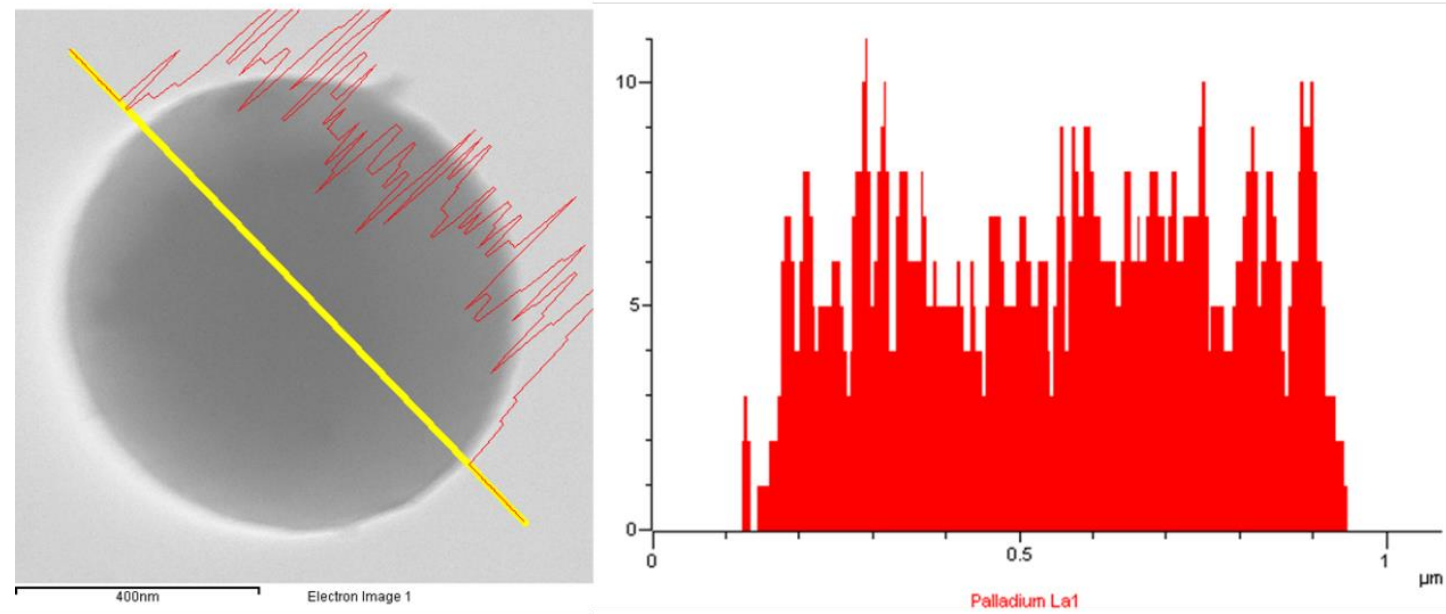

Figure S20. EDX line scan of Pd for spent MPOS-Pd catalyst after 5 cycles of Suzuki-Miyaura reaction at $50{ }^{\circ} \mathrm{C}$ for $60 \mathrm{~min}$.
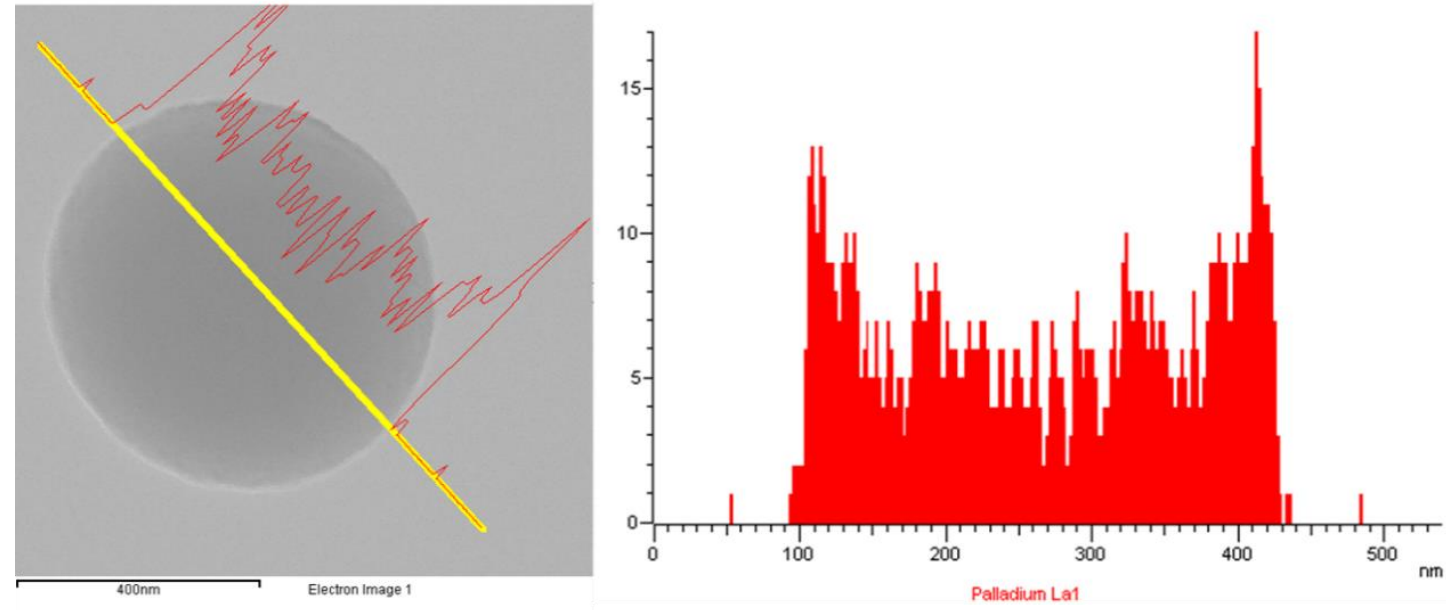

Figure S21. EDX line scan of Pd for a spent MPOS-Pd sphere after regeneration. 


\section{Supporting Tables}

Table S1. Detailed Suzuki-Miyaura reaction rate between aryl iodides and phenylboronic acid using MPOS-Pd catalyst at $80{ }^{\circ} \mathrm{C}$.

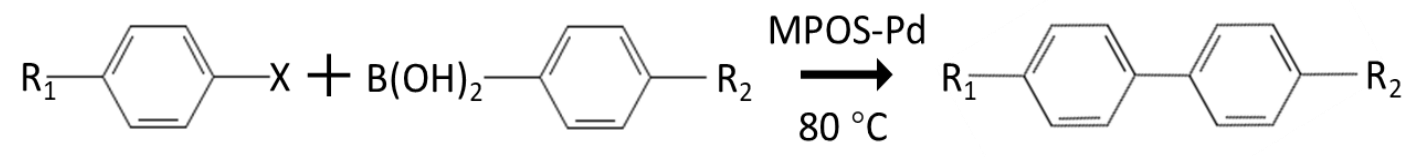

\begin{tabular}{|c|c|c|c|c|c|c|}
\hline Entry & $\mathbf{R}_{1}$ & $\mathbf{X}$ & $\mathbf{R}_{2}$ & Time / min & Conversion / \% & TOF $/ \mathrm{h}^{-1}$ \\
\hline \multirow{5}{*}{1} & \multirow{5}{*}{$-\mathrm{H}$} & \multirow{5}{*}{ I } & \multirow{5}{*}{$-\mathrm{H}$} & 0.5 & 49.9 & 7095 \\
\hline & & & & 10 & 88.7 & \\
\hline & & & & & & \\
\hline & & & & 15 & 96.3 & \\
\hline & & & & 30 & 98.4 & \\
\hline \multirow{3}{*}{2} & \multirow{3}{*}{$-\mathrm{CH}_{3}$} & \multirow{3}{*}{ I } & \multirow{3}{*}{$-\mathrm{H}$} & 5 & 64.5 & 457 \\
\hline & & & & 15 & 95.0 & \\
\hline & & & & 30 & 98.7 & \\
\hline \multirow{3}{*}{3} & \multirow{3}{*}{$-\mathrm{OCH}_{3}$} & \multirow{3}{*}{ I } & \multirow{3}{*}{$-\mathrm{H}$} & 5 & 15.1 & 107 \\
\hline & & & & 15 & 86.3 & \\
\hline & & & & 30 & 98.2 & \\
\hline
\end{tabular}


Table S2. Detailed Suzuki-Miyaura reaction rate between aryl iodides and biphenylboronic acid using MPOS-Pd catalyst at $80^{\circ} \mathrm{C}$.

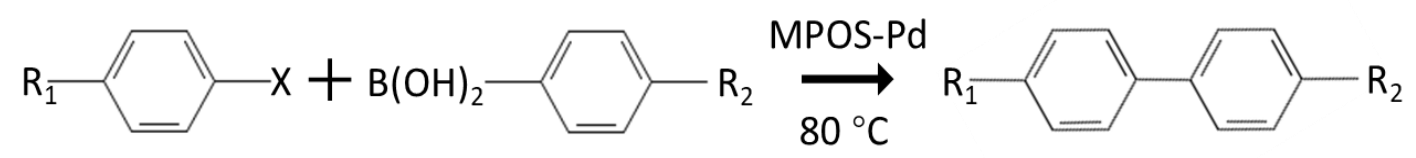

\begin{tabular}{|c|c|c|c|c|c|c|}
\hline Entry & $\mathbf{R}_{1}$ & $\mathbf{X}$ & $\mathbf{R}_{2}$ & Time / min & Conversion / \% & $\mathrm{TOF} / \mathrm{h}^{-1}$ \\
\hline \multirow{5}{*}{1} & \multirow{5}{*}{$-\mathrm{H}$} & \multirow{5}{*}{ I } & \multirow{5}{*}{$-\mathrm{Ph}$} & 0.5 & 42.2 & 5988 \\
\hline & & & & 5 & 74.9 & \\
\hline & & & & & & \\
\hline & & & & 15 & 95.3 & \\
\hline & & & & 30 & 100 & \\
\hline \multirow{5}{*}{2} & \multirow{5}{*}{$-\mathrm{CH}_{3}$} & \multirow{5}{*}{ I } & \multirow{5}{*}{$-\mathrm{Ph}$} & 0.5 & 21.0 & 2980 \\
\hline & & & & 5 & 74.2 & \\
\hline & & & & & & \\
\hline & & & & 15 & 92.1 & \\
\hline & & & & 30 & 100 & \\
\hline \multirow{5}{*}{3} & \multirow{5}{*}{$-\mathrm{OCH}_{3}$} & \multirow{5}{*}{ I } & \multirow{5}{*}{$-\mathrm{Ph}$} & 0.5 & 10.9 & 1547 \\
\hline & & & & 5 & 61.3 & \\
\hline & & & & & & \\
\hline & & & & 15 & 97.7 & \\
\hline & & & & 30 & 100 & \\
\hline
\end{tabular}


Table S3. Detailed Suzuki-Miyaura reaction rate between aryl bromides and phenylboronic acid using MPOS-Pd catalyst at $80^{\circ} \mathrm{C}$.

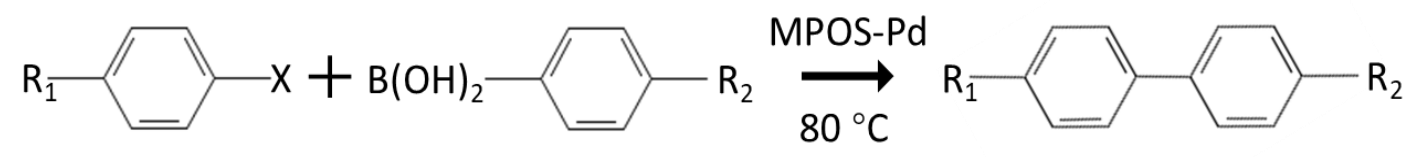

\begin{tabular}{|c|c|c|c|c|c|c|}
\hline Entry & $\mathbf{R}_{1}$ & $\mathbf{X}$ & $\mathbf{R}_{2}$ & Time / min & Conversion / \% & $\mathrm{TOF} / \mathrm{h}^{-1}$ \\
\hline \multirow{5}{*}{1} & \multirow{5}{*}{$-\mathrm{H}$} & \multirow{5}{*}{$\mathrm{Br}$} & \multirow{5}{*}{$-\mathrm{H}$} & 0.5 & 12.5 & 1774 \\
\hline & & & & 5 & 22.5 & \\
\hline & & & & 15 & 29.5 & \\
\hline & & & & 30 & 32.6 & \\
\hline & & & & 60 & 33.3 & \\
\hline \multirow{3}{*}{2} & \multirow{3}{*}{$-\mathrm{OCH}_{3}$} & \multirow{3}{*}{$\mathrm{Br}$} & \multirow{3}{*}{$-\mathrm{H}$} & 15 & 6.3 & 30 \\
\hline & & & & 30 & 10.4 & \\
\hline & & & & 60 & 11.5 & \\
\hline
\end{tabular}


Table S4. Detailed Suzuki-Miyaura reaction rate between iodobenzene and phenylboronic acid using MPOS-Pd catalyst at $80{ }^{\circ} \mathrm{C}$. The reaction mixture was filtered after 4 min of reaction and the hot filtrate was transferred to a new round bottom flask with $1 \mathrm{mmol}$ of $\mathrm{K}_{2} \mathrm{CO}_{3}$ to continue the reaction.

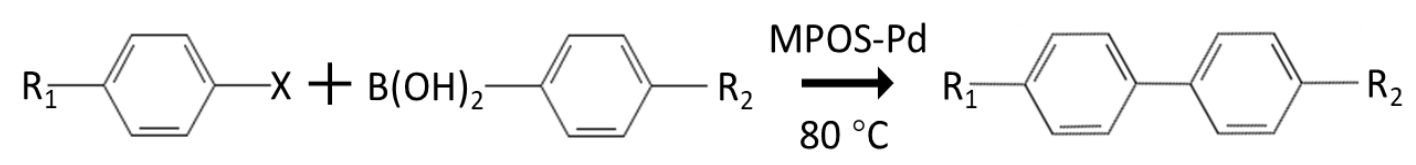

\begin{tabular}{|c|c|c|c|c|c|c|}
\hline Entry & $\mathbf{R}_{1}$ & $\mathbf{X}$ & $\mathbf{R}_{2}$ & Time / min & Conversion / \% & TOF $/ h^{-1}$ \\
\hline \multirow{5}{*}{1} & \multirow{5}{*}{$-\mathrm{H}$} & \multirow{5}{*}{ I } & \multirow{5}{*}{$-\mathrm{H}$} & 4 & 64.4 & N.A. \\
\hline & & & & 10 & 87.5 & \\
\hline & & & & & & \\
\hline & & & & 20 & 95.5 & \\
\hline & & & & 30 & 96.9 & \\
\hline
\end{tabular}


Table S5. Detailed Suzuki-Miyaura reaction rate between iodobenzene and phenylboronic acid using MPOS-Pd catalyst at $50{ }^{\circ} \mathrm{C}$ and room temperature $\left(23{ }^{\circ} \mathrm{C}\right)$.

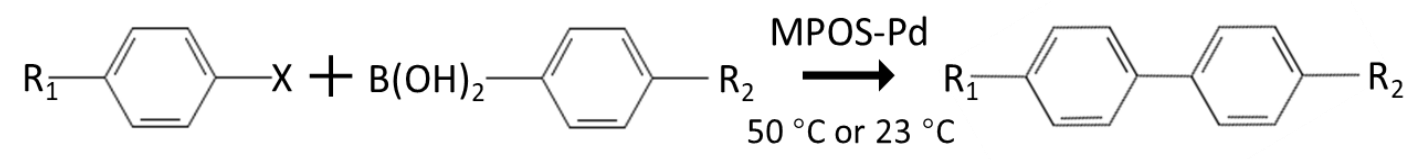

\begin{tabular}{|c|c|c|c|c|c|c|}
\hline Temp. $/{ }^{\circ} \mathrm{C}$ & $\mathbf{R}_{1}$ & $\mathbf{X}$ & $\mathbf{R}_{2}$ & Time / min & Conversion / \% & TOF $/ h^{-1}$ \\
\hline \multirow{4}{*}{50} & \multirow{4}{*}{$-\mathrm{H}$} & \multirow{4}{*}{ I } & \multirow{4}{*}{$-\mathrm{H}$} & 0.5 & 11.1 & 1578 \\
\hline & & & & 5 & 58.2 & \\
\hline & & & & 15 & 82.2 & \\
\hline & & & & 30 & 92.7 & \\
\hline \multirow{4}{*}{23} & \multirow{4}{*}{$-\mathrm{H}$} & \multirow{4}{*}{ I } & \multirow{4}{*}{$-\mathrm{H}$} & 5 & 13.1 & 186 \\
\hline & & & & 15 & 29.5 & \\
\hline & & & & 30 & 37.6 & \\
\hline & & & & 60 & 56.4 & \\
\hline
\end{tabular}


Table S6. Iodobenzene conversion and Pd loading of catalyst for 5 repeated cycles of SuzukiMiyaura reaction at $80{ }^{\circ} \mathrm{C}$ for $30 \mathrm{~min}$.

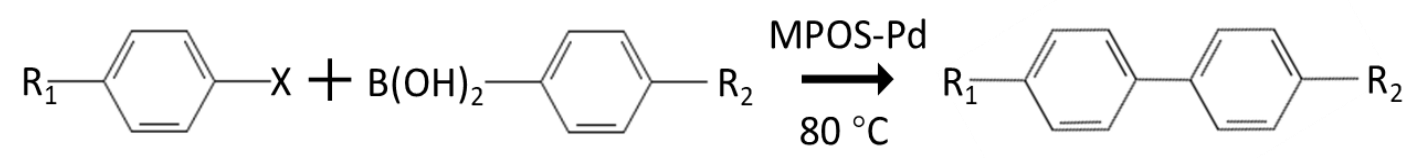

\begin{tabular}{|c|c|c|c|c|c|c|}
\hline Catalyst & $\mathbf{R}_{\mathbf{1}}$ & $\mathbf{X}$ & $\mathbf{R}_{\mathbf{2}}$ & Run & Conversion / \% & Pd loading / wt \% \\
\hline & & & 1 & 98.5 & 1.6 \\
\hline & & & & & & \\
\hline & & & & & & \\
\hline & & & & & & \\
\hline
\end{tabular}


Table S7. Iodobenzene conversion and Pd loading of catalyst for 5 repeated cycles of SuzukiMiyaura reaction at $80{ }^{\circ} \mathrm{C}$ for $60 \mathrm{~min}$.

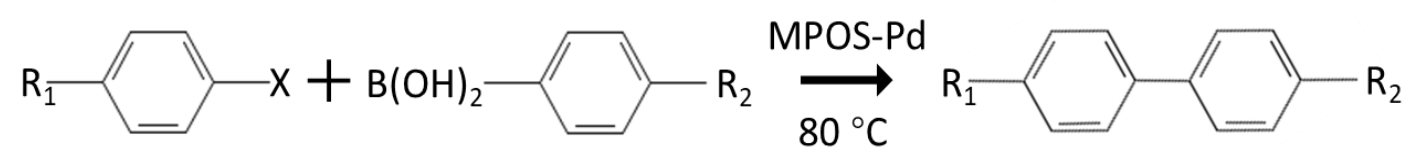

\begin{tabular}{|c|c|c|c|c|c|c|}
\hline Catalyst & $\mathbf{R}_{1}$ & $\mathbf{X}$ & $\mathbf{R}_{\mathbf{2}}$ & Run & Conversion / \% & Pd loading / wt \% \\
\hline \multirow{5}{*}{ MPOS-Pd } & \multirow{5}{*}{$-\mathrm{H}$} & \multirow{5}{*}{ I } & \multirow{5}{*}{$-\mathrm{H}$} & 1 & 100 & \\
\hline & & & & 2 & 100 & \\
\hline & & & & 3 & 100 & \\
\hline & & & & 4 & 100 & \\
\hline & & & & 5 & 100 & 1.7 \\
\hline
\end{tabular}


Table S8. Iodobenzene conversion and Pd loading of catalyst for 5 repeated cycles of SuzukiMiyaura reaction at $50{ }^{\circ} \mathrm{C}$ for $60 \mathrm{~min}$.

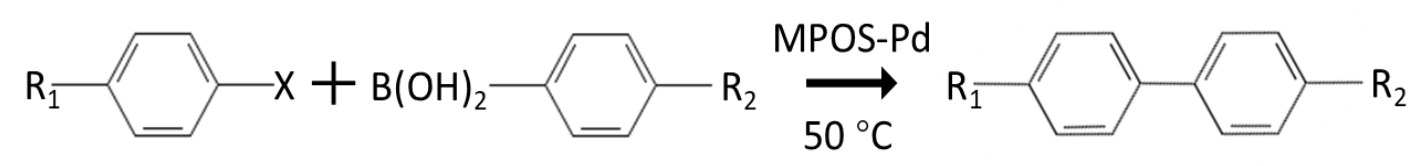

\begin{tabular}{|c|c|c|c|c|c|c|}
\hline Catalyst & $\mathbf{R}_{1}$ & $\mathbf{X}$ & $\mathbf{R}_{\mathbf{2}}$ & Run & Conversion / \% & Pd loading / wt \% \\
\hline \multirow{5}{*}{ MPOS-Pd } & \multirow{5}{*}{$-\mathrm{H}$} & \multirow{5}{*}{ I } & \multirow{5}{*}{$-\mathrm{H}$} & 1 & 93.0 & \\
\hline & & & & 2 & 93.7 & \\
\hline & & & & 3 & 85.5 & \\
\hline & & & & 4 & 57.7 & \\
\hline & & & & 5 & 40.1 & 1.3 \\
\hline
\end{tabular}

